\title{
Antiplatelet Therapy with Cangrelor in Patients Undergoing Surgery after Coronary Stent Implantation: A Real-World Bridging Protocol Experience
}

\author{
Roberta Rossini $^{1, *}$ Giulia Masiero ${ }^{1, *(1)}$ Claudia Fruttero $^{1}$ Enrico Passamonti ${ }^{2}$ Elba Calvaruso ${ }^{2}$ \\ Moreno Cecconi ${ }^{3}$ Cesare Carlucci ${ }^{3}$ Marco Mojoli ${ }^{4}$ Parodi Guido $^{5}$ Giuseppe Talanas ${ }^{5}$ \\ Simona Pierini ${ }^{6}$ Paolo Canova ${ }^{7} \quad$ Nicoletta De Cesare ${ }^{8} \quad$ Stefania Luceri $^{9} \quad$ Nicoletta Barzaghi ${ }^{1}$ \\ Giulio Melloni ${ }^{1}$ Giorgio Baralis ${ }^{1}$ Alessandro Locatelli ${ }^{1}$ Giuseppe Musumeci ${ }^{1}$ Dominick J. Angiolillo ${ }^{10}$
}

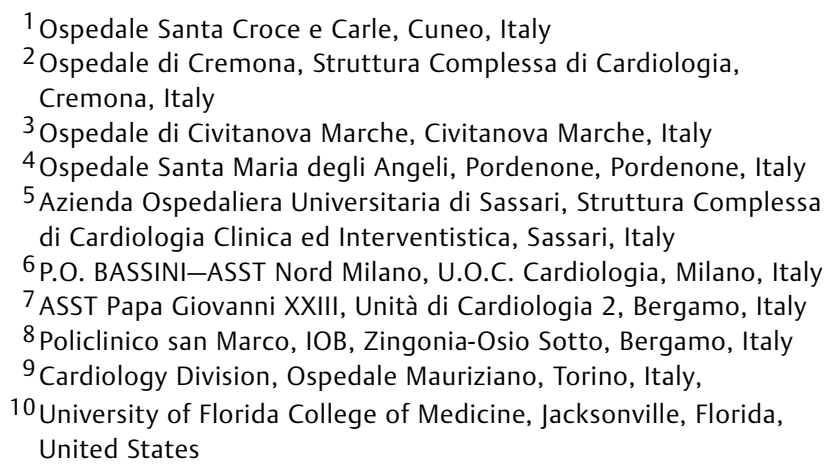

Address for correspondence Roberta Rossini, MD, PhD, Dipartimento Cardiovascolare, Ospedale S. Croce e Carle, Cuneo 12100, Italy (e-mail: roberta.rossini2@gmail.com).

TH Open 2020;4:e437-e445.

\begin{abstract}
Keywords

- antiplatelet therapy

- stent

- surgery

- bridging

- cangrelor

Objective The aim of the study is to describe the real-world use of the $\mathrm{P} 2 \mathrm{Y}_{12}$ inhibitor cangrelor as a bridging strategy in patients at high thrombotic risk after percutaneous coronary intervention $(\mathrm{PCl})$ and referred to surgery requiring perioperative withdrawal of dual antiplatelet therapy (DAPT).

Materials and Methods We collected data from nine Italian centers on patients with previous PCI who were still on DAPT and undergoing nondeferrable surgery requiring DAPT discontinuation. A perioperative standardized bridging protocol with cangrelor was used. Results Between December 2017 and April 2019, 24 patients (mean age 72 years; male $79 \%$ ) were enrolled. All patients were at high thrombotic risk after $\mathrm{PCl}$ and required nondeferrable intermediate to high bleeding risk surgery requiring DAPT discontinuation ( $4.6 \pm 1.7$ days). Cangrelor infusion was started at a bridging dose $(0.75 \mu \mathrm{g} / \mathrm{kg} / \mathrm{min}) 3$ days before planned surgery and was discontinued $6.6 \pm 1.5$ hours prior to surgical incision. In $55 \%$ of patients, cangrelor was resumed at $9 \pm 6$ hours following surgery for a mean of $39 \pm 38$ hours. One cardiac death was reported after 3 hours of cangrelor discontinuation prior to surgery. No ischemic outcomes occurred after surgery and up to 30-days follow-up. The mean hemoglobin drop was $<2 \mathrm{~g} / \mathrm{dL}$; nine patients received blood transfusions consistent with the type of surgery, but no life-threatening or fatal bleeding occurred.
\end{abstract}

These authors contributed equally to this work.

received

October 16, 2019

accepted after revision

November 2, 2020
DOI https://doi.org/

10.1055/s-0040-1721504. ISSN 2512-9465. (c) 2020 Georg Thieme Verlag KG Rüdigerstraße 14, 70469 Stuttgart, Germany
License terms

(c) (i) 
Conclusion Perioperative bridging therapy with cangrelor is a feasible approach for stented patients at high thrombotic risk and referred to surgery requiring DAPT discontinuation. Larger studies are warranted to support the safety of this strategy.

\section{Introduction}

Dual antiplatelet therapy (DAPT) with aspirin and a $\mathrm{P}_{2} \mathrm{Y}_{12}$ inhibitor is the standard of care to prevent thrombotic complications in patients undergoing percutaneous coronary intervention ( $\mathrm{PCI}$ ) with stent implantation. ${ }^{1}$ Perioperative management of DAPT in patients undergoing nondeferrable surgery still raises relevant safety concerns. On one hand, discontinuation of DAPT to reduce bleeding complications is associated with an enhanced risk of thrombotic events, while maintenance of DAPT to avoid perioperative thrombotic complications increases the risk of bleeding and the need for transfusions, which are both known determinants of poor prognosis. ${ }^{2}$ A strategy of temporary transition with an intravenous antiplatelet agent may represent a desirable treatment option in patients deemed at high thrombotic risk undergoing nondeferrable relevant bleeding risk surgery requiring a predictable interruption of platelet inhibition. $^{3-6}$ The European Society of Cardiology (ESC) guidelines recommend a bridging strategy with an intravenous antiplatelet agent if both oral antiplatelet agents have to be discontinued perioperatively, especially within 1 month after $\mathrm{PCI} .^{5}$ However, currently there are no antiplatelet agents approved by drug regulating agencies for such bridging indication.

Cangrelor is an intravenous, rapidly acting, and reversible potent $\mathrm{P}_{2} \mathrm{Y}_{12}$ platelet receptor antagonist approved for the reduction of thrombotic cardiovascular events in $\mathrm{P}_{2} \mathrm{Y}_{12}$ inhibitor naïve patients undergoing $\mathrm{PCI}^{7,8}$ Cangrelor also has a very short half-life (3-6 minutes) with an offset of its antiplatelet effects within 60 minutes making it an attractive option for bridging therapy. Accordingly, a dose-finding investigation was conducted to identify a regimen of cangrelor associated with a "thienopyridine-like" effect for bridging purposes, hence not as potent as that used for its $\mathrm{PCI}$ indication which is known to lead to near complete P2Y 12 inhibition. Such identified bridging dosing regimen of cangrelor $(0.75 \mu \mathrm{g} / \mathrm{kg} / \mathrm{min})$ was tested in a prospective randomized double-blind study among thienopyridine-treated patients undergoing coronary artery bypass grafting surgery (CABG) showing to achieve adequate levels of platelet inhibition during the washout phase from oral $\mathrm{P}_{2} \mathrm{Y}_{12}$ inhibitors until the time of surgery without safety (bleeding or nonbleeding) concerns. ${ }^{9}$ However, there is limited reported data on the use of cangrelor among patients undergoing noncardiac surgery (NCS). ${ }^{10-16}$ On this background, we report the results of a prospective, multicenter registry describing realworld experience of a prespecified bridging protocol using cangrelor conducted in patients referred to nondeferrable intermediate to high bleeding risk surgery requiring withdrawal of DAPT.

\section{Materials and Methods}

\section{Study Population}

We prospectively collected data from nine Italian centers that had included patients undergoing surgery using cangrelor as a bridging strategy in the perioperative phase (-Table 1 ). All patients were still on DAPT due to recent coronary stent implantation and required nondeferrable, intermediate to high bleeding risk surgery demanding discontinuation of one or both antiplatelet agents. Patients with prohibitive hemorrhagic risk profile or active bleeding were not considered suitable for bridging therapy with cangrelor. Guidelines indicate that the perioperative management of antithrombotic therapy needs to be defined in a multidisciplinary manner to better state the trade-off between ischemia and bleeding..$^{17-19}$ To predict the individual risk of thrombotic complications against the anticipated risk of surgical bleeding complications, a multidisciplinary consensus document among cardiologists, surgeons, and anesthesiologists on practical recommendations for standardizing management of antithrombotic therapy management in patients treated with coronary stents (Surgery After Stenting 2) was used (https://itunes.apple.com/us/app/stentsurgery/id551350096?mt1/48). ${ }^{20,21}$ Briefly, the document defined the thrombotic risk (low, intermediate, or high) by combining angiographic and clinical features, time from stent implantation, type of implanted stent, and perioperative need for DAPT discontinuation. Moreover, surgical bleeding risk was defined into low, intermediate, or high according to the inherent hemorrhagic risk of over 250 cardiac and noncardiac surgical procedures based on the amount of blood loss and the anticipated difficulty in achieving adequate local hemostasis. ${ }^{20,21}$

\section{Bridging Protocol}

A standardized bridging protocol using cangrelor infusion before and eventually after surgery was reserved for patients deemed at high thrombotic risk undergoing nondeferrable surgery at intermediate to high risk of bleeding, which requires a predictable interruption of platelet inhibition at the time of surgery ${ }^{21,22}$ (- Fig. 1). According to International Guidelines, clopidogrel and ticagrelor were discontinued for 5 days before surgery, while prasugrel was discontinued for 7 days. ${ }^{5}$ In line with expert consensus recommendation, cangrelor at a bridging dose regimen $(0.75 \mu \mathrm{g} / \mathrm{kg} / \mathrm{min}$ infusion without a bolus) was initiated 2 to 3 days following clopidogrel and ticagrelor discontinuation and 3 to 4 days after prasugrel discontinuation. ${ }^{9,22}$ As a matter of fact, previous data reported a broad variability in platelet reactivity after discontinuation of thienopyridine therapy, meaning that a considerable number of patients are not adequately protected when stopping thienopyridine therapy for up to a week. ${ }^{9}$ Cangrelor infusion was discontinued up 1 to 10 hours 


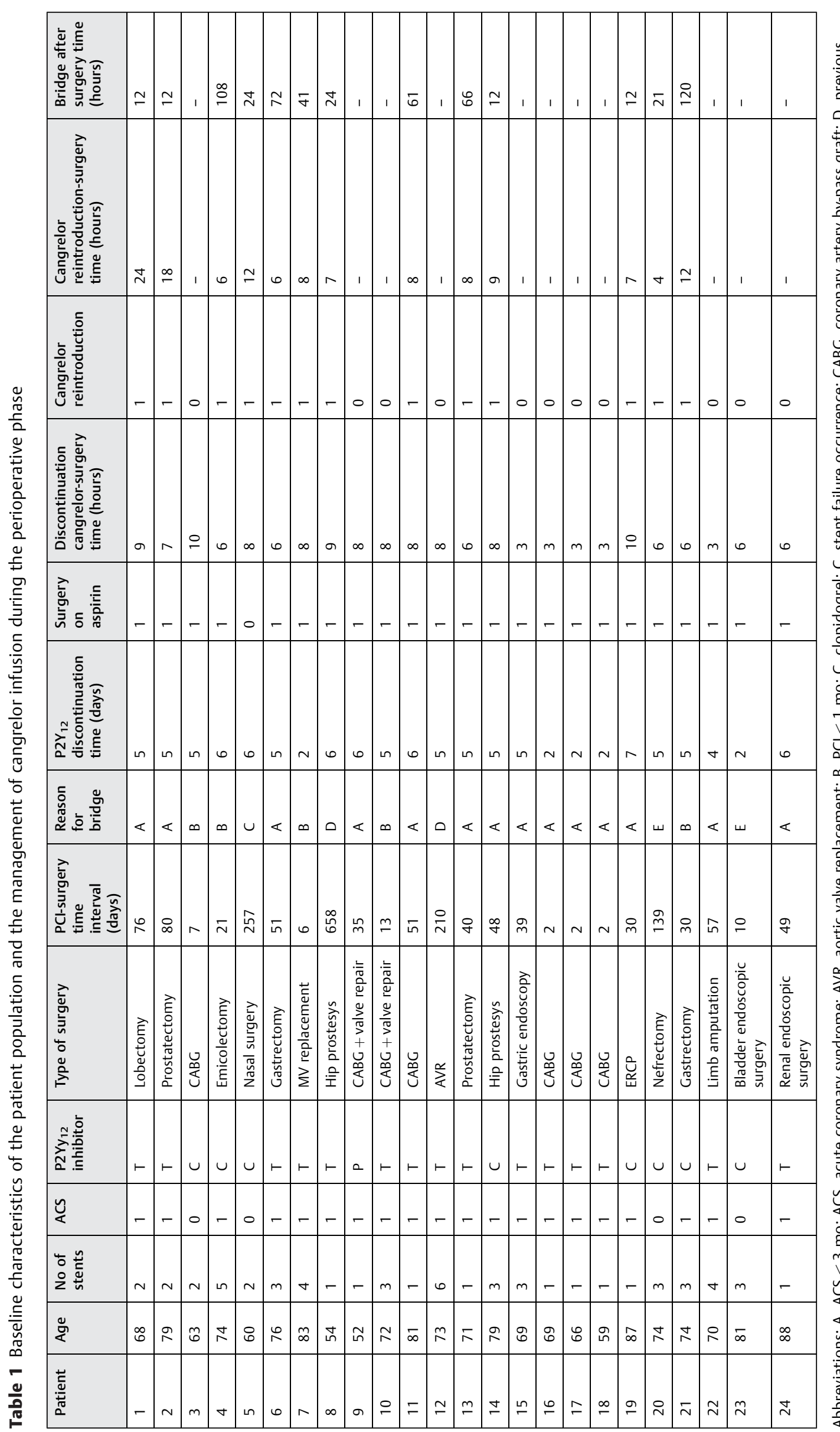

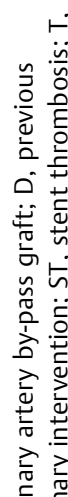

离交

ن

巡苛

产

䓀

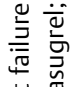

若蓄

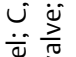

它 $\frac{\pi}{\frac{0}{n}}$

$u$

है

$\overline{\mathrm{V}}$

$\overline{\mathrm{D}}$ 苋

si

苞 음

음

弯

는 음 艺苍 है ข 는 ¿ิ

产 $\frac{x}{0}$

它

ن்

造

它 壳

V $\bar{E}$

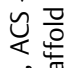

$<$

븡 음 


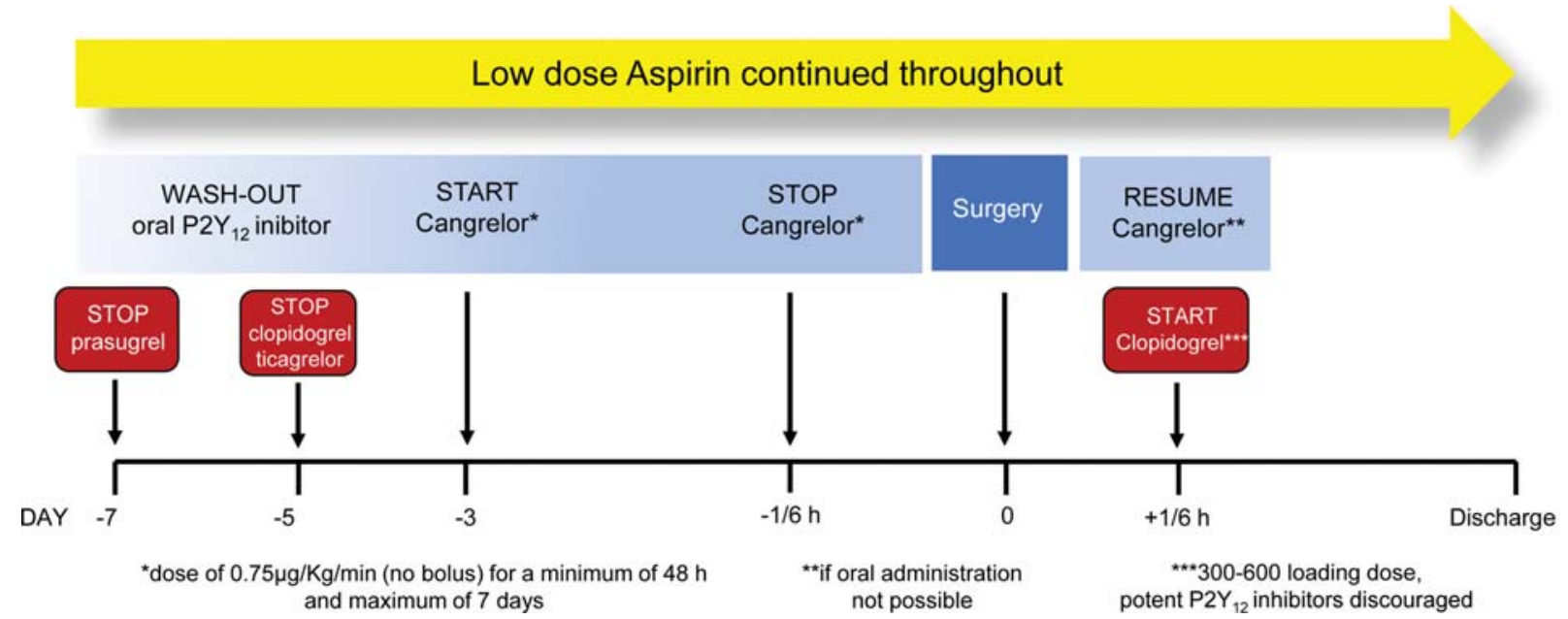

Fig. 1 Standardized bridging protocol using cangrelor infusion before and eventually after nondeferrable surgery which required discontinuation of one or both antiplatelet agents in patients with previous $\mathrm{PCl} .{ }^{21} \mathrm{PCI}$, percutaneous coronary intervention.

before surgery. Otherwise, according to BRIDGE Trial design, a lower $\mathrm{P}_{2} \mathrm{Y}_{12}$ inhibitor discontinuation time and PCI-tosurgery time intervals were allowed in patients referred to nonemergent $C A B G$ planned between 48 hours but no longer than 7 days following coronary stent implantation. ${ }^{9}$ Since thrombotic complications occur most frequently soon after surgery, close clinical and electrocardiographic monitoring in an intensive care unit was emphasized in the postoperative period. Complete blood count assessments were performed daily to monitor hemoglobin levels. Once successful hemostasis was achieved, oral $\mathrm{P}_{2} \mathrm{Y}_{12}$ inhibiting therapy was resumed within 24 to 48 hours. Clopidogrel was preferred over prasugrel or ticagrelor in this setting of increased bleeding risk patients. In particular, to avoid a potential drug-drug interaction with cangrelor, clopidogrel $600 \mathrm{mg}$ loading dose (LD) was administered immediately after discontinuation of cangrelor. ${ }^{22,23}$ If oral $\mathrm{P}_{2} \mathrm{Y}_{12}$ inhibiting therapy was temporarily not administrable (i.e., in case of failed gastrointestinal function recovery or prolonged maintenance of drainages), intravenous infusion of cangrelor was resumed, after careful evaluation of the bleeding risk.

\section{Study End Points}

Clinical events during the perioperative phase (up to 48 hours from surgery) and at 30-days follow-up were prospectively collected. Cardiac death, periprocedural and spontaneous myocardial infarction (MI), urgent target-lesion revascularization (TLR), and definite stent thrombosis (ST) were assessed according to the Academic Research Consortium Criteria and the American College of Cardiology/American Heart Association (ACC/AHA) cardiovascular end points data standards. ${ }^{24}$ Major ischemic adverse cardiac events (MACE) were defined as the composite of cardiac death, MI, and TLR.

Bleeding events were defined using the Global Use of Strategies to Open Occluded Coronary Arteries (GUSTO) and Bleeding Academic Research Consortium (BARC) definition. ${ }^{25}$ Major bleedings were defined as GUSTO-defined severe/life-threatening bleedings and the composite of
BARC-defined type 3b, type 3c, and type 5 bleedings. For the purpose of the study, CABG-related bleeding (BARC type 4) was included in the major bleeding category in case of fatal bleeding, reoperation following closure of sternotomy for the purpose of controlling bleeding, transfusion of $\geq 5$ units or relevant chest tube output. Mild bleedings were defined as GUSTO-defined moderate bleedings and BARCdefined type 3a. The amount of blood loss from drainages, the hemoglobin drops, and the need for blood transfusion were also assessed.

\section{Statistical Analysis}

All efficacy and safety end points were collected during the perioperative phase (evaluated within 48 hours from surgery) and at 30 days follow-up. Quantitative variables were summarized as mean \pm standard deviation, while categorical ones as count and percentages in each category.

\section{Results}

\section{Patient Characteristics and Perioperative Management}

Between December 2017 and April 2019, 24 patients were identified to be at high thrombotic risk and required nondeferrable intermediate-high bleeding risk surgery. Baseline characteristics of the patient population and data on cangrelor infusion during the perioperative phase are shown in - Table 1. Mean age was $72 \pm 9$ years and $79 \%$ were men. In the majority ( $83 \%$ ) of patients, the index $\mathrm{PCI}$ was performed due to an acute coronary syndrome (ACS) and $2.1 \pm 1.5$ stents per patient were implanted. The average time from $\mathrm{PCI}$ to surgery was $80 \pm 136$ days. Two-thirds of the patients were on ticagrelor $90 \mathrm{mg}$ bid ( $n=14,58 \%$ of cases), one-third on clopidogrel ( $n=9,38 \%$ of the cases), and a minority on prasugrel ( $n=1,4 \%$ of cases). All patients were also on aspirin $100 \mathrm{mg} /$ qd. According to a multidisciplinary evaluation and SAS2 criteria, ${ }^{20}$ high thrombotic risk categories included: PCI within 1 month (patients number 3-4-7-10-21), ACS within 3 months (patients number 1-2-6-9-11-13 to 19-22-24), prior stent failure due to definite subacute ST 


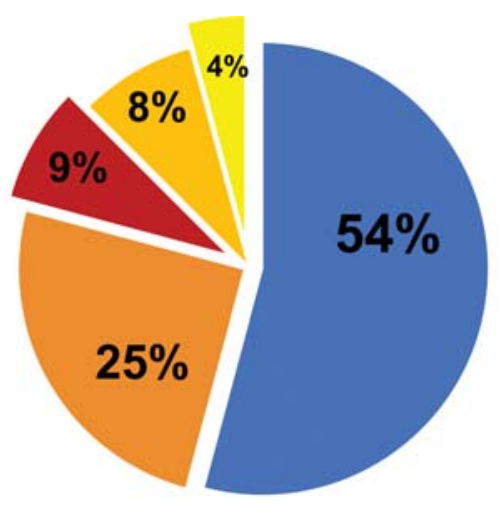

- SCA $<3$ months

= $\mathrm{PCl}<1$ month

- Stent failure

- Complex $\mathrm{PCl}$ BVS

Fig. 2 High thrombotic risk categories included in the studied patient population undergoing to bridging with cangrelor for nondeferrable relevant bleeding risk surgery.

(patient number 5), previous complex PCI with multiple stents implantation and left main involvement (patients number 2023), and use of a bioresorbable vascular scaffold (BRS) (patients number 8-12) (-Fig. 2). Intermediate to high bleeding risk surgeries, deemed nondeferrable from a surgical point-ofview especially due to recently encountered neoplastic pathology, included: pulmonary lobectomy (patient number 1), prostatectomy (patients number 2-13), colectomy (patient number 4), endoscopic surgery (patients number 15-2324), gastrectomy (patients number 6-21), nephrectomy (patient number 20), paranasal sinus surgery (patient number 5), hip replacement (patients number 8-14), endoscopy sphincterotomy (patient number 19), and limb amputation (patient number 22) (-Fig. 3). Eight patients (numbers 3-7-9 to 12-16 to 18) were planned for nonemergent CABG and valvular repair. The average time of $\mathrm{P}_{2} \mathrm{Y}_{12}$ inhibitor discontinuation was $4.5 \pm 1.7$ days prior to surgery. All patients but one maintained aspirin through the perioperative phase. Cangrelor infusion was started at the bridging dose $(0.75 \mu \mathrm{g} / \mathrm{kg} / \mathrm{min})$ $2.9 \pm 0.9$ days before planned surgery and was discontinued $6.6 \pm 1.5$ hours prior to surgical incision. After surgery, drainages were left in all but six patients. In $55 \%$ of patients, cangrelor was resumed within 24 hours from surgery (mean time $8.6 \pm 6.1$ hours) for a mean of $39 \pm 38$ hours. Drainages were removed after discontinuation of cangrelor to reduce bleeding complications. Within 2 hours from postoperative cangrelor discontinuation, a $600 \mathrm{mg}$ clopidogrel LD was administered in all patients. In all other patients, clopidogrel was resumed at $36 \pm 22$ hours after surgery once successful hemostasis was achieved.

\section{Outcomes}

Adverse events are reported in - Table 2. Prior to surgery, one cardiac death occurred due to fatal ST elevation MI at 3 hours after cangrelor discontinuation. The patient was still on aspirin $100 \mathrm{mg} / \mathrm{qd}$, whereas ticagrelor $90 \mathrm{mg} /$ bid had been discontinued 5 days prior to nonemergent surgery. This 70year-old female patient was afflicted by chronic limb-threatening ischemia (CLTI) with lower extremities rest pain (Radford stage IV). She experienced a failed attempt of revascularization complicated with an ACS treated with an effective complex PCI (four stents implanted) on the left main and left descending artery. Due to progression of CLTI disease to incapacitating pain and nonhealing wounds (Radford stage V) but with no signs of infection she was scheduled for a nonemergent but nondeferrable lower limb amputation 57 days after the $\mathrm{PCI} .^{26}$ No other major ischemic adverse

$50 \%$

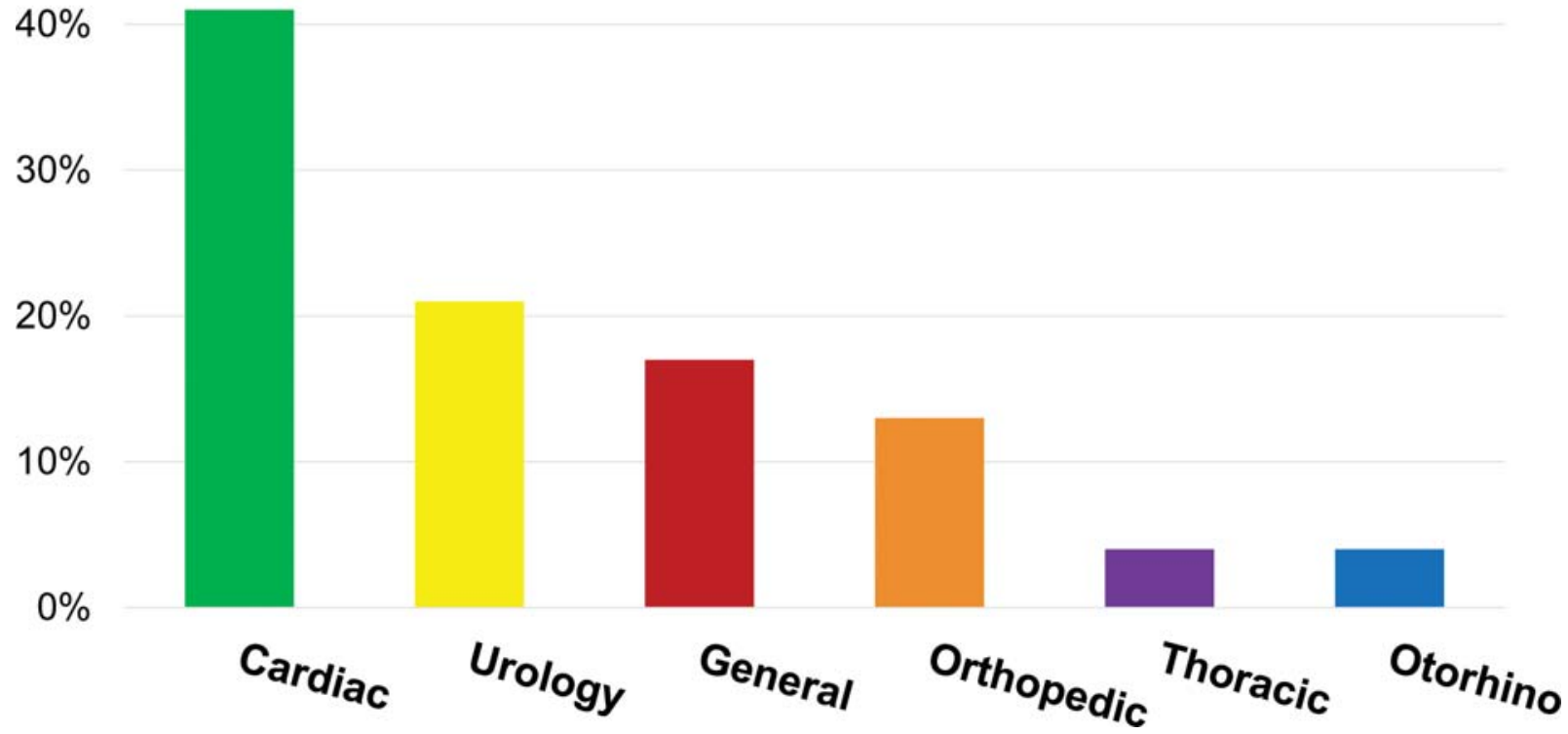

Fig. 3 Nondeferrable, intermediate-high bleeding risk surgeries included in the studied patient population undergoing bridging with cangrelor for high ischemic risk. 
Table 2 Clinical ischemic and bleeding events during the perioperative phase (up to $48 \mathrm{~h}$ from surgery) and at $30-\mathrm{d}$ follow-up

\begin{tabular}{|c|c|c|}
\hline & $<48 \mathrm{~h}$ & $>48 \mathrm{~h}$ to $30 \mathrm{~d}$ \\
\hline \multicolumn{3}{|l|}{ Ischemic events } \\
\hline MACE & $1(4 \%)$ & $0(0 \%)$ \\
\hline Cardiac death & $1(4 \%)$ & $0(0 \%)$ \\
\hline MI & $1(4 \%)$ & $0(0 \%)$ \\
\hline Urgent TLR & $0(0 \%)$ & $0(0 \%)$ \\
\hline ST & $1(4 \%)$ & $0(0 \%)$ \\
\hline \multicolumn{3}{|l|}{ Bleeding events } \\
\hline $\begin{array}{l}\text { GUSTO-defined severe/ } \\
\text { life-threatening }\end{array}$ & $0(\%)$ & $0(\%)$ \\
\hline BARC-defined type 5 & $0(\%)$ & $0(\%)$ \\
\hline GUSTO-defined moderate & $9(38 \%)$ & $4(17 \%)$ \\
\hline BARC-defined type $3 a$ & $9(38 \%)$ & $4(17 \%)$ \\
\hline BARC-defined type 3b-3c & $3(13 \%)$ & $2(8 \%)$ \\
\hline Hemoglobin drop $(\mathrm{g} / \mathrm{dL})$ & $1.8 \pm 1.7$ & - \\
\hline Need for blood transfusion & $9(38 \%)$ & $4(17 \%)$ \\
\hline
\end{tabular}

Abbreviations: BARC, Bleeding Academic Research Consortium; GUSTO, Global Use of Strategies to Open Occluded Coronary Arteries; MACE, major adverse ischemic events; MI, myocardial infarction; ST, stent thrombosis; TLR, target lesion revascularization.

Note: Variables are presented as count and percentages or mean values \pm standard deviation.

outcomes occurred up to 30 days follow-up. The average hemoglobin drop was $1.8 \pm 1.8 \mathrm{~g} / \mathrm{dL}$ and nine patients required periprocedural blood transfusions which was consistent with the type of surgery performed, known to need a larger spending of blood products, as orthopaedic, general, and cardiac surgery. ${ }^{27}$ One-third of patients experienced periprocedural BARC 3/GUSTO moderate bleeding events due to drainages output, but a hemoglobin drop $\geq 3 \mathrm{~g} / \mathrm{dL}$ was observed in only three cases. No fatal, life-threatening, or intracranial bleedings occurred. No patient required reoperation or experienced hemodynamic compromise due to bleeding.

\section{Discussion}

To the best of our knowledge, this is the largest multicenter experience on a bridging protocol with cangrelor in stented patients at high ischemic risk undergoing nondeferrable intermediate-high bleeding risk surgery. In the present case series, a reassuring safety bleeding profile of cangrelor was demonstrated, with no identified fatal/life-threatening bleedings or major blood loss requiring re-operation occurring in the perioperative phase.

Compared with patients without CAD, those who have previous $\mathrm{PCI}$ are at higher risk for MACE when undergoing surgery. ${ }^{6,28,29}$ The risk of an ischemic event (e.g., ST, MI, and cardiac death) associated with surgery is strictly dependent on time from PCI, patient's surgical and cardiac risk, and the need for DAPT interruption. ${ }^{2-4}$ Moreover, surgery itself is associated with proinflammatory and prothrombotic effects regardless of previous stenting. ${ }^{2-4}$ Several observational studies have reported that the surgical risk in PCI treated patients stabilizes after 3 to 6 months; furthermore, selected patients without high-risk clinical or lesion characteristics showed the same perioperative risk of patients without CAD already beyond the first month after any type of stent implantation. ${ }^{21,30}$ In fact, current ESC guidelines recommend $\mathrm{P}_{2} \mathrm{Y}_{12}$ inhibitor discontinuation 1 month after PCI, irrespective of the stent type, if aspirin can be maintained throughout the perioperative period. ${ }^{5}$ In patients with recent MI or other high ischemic risk features requiring DAPT, elective surgery should be postponed for up to 6 months. ${ }^{5} \mathrm{~A}$ bridging strategy with an intravenous antiplatelet agent is required if both oral antiplatelet agents have to be discontinued perioperatively, especially within 1 month after PCI. ${ }^{5}$ However, there are no specific recommendations on perioperative antiplatelet therapy provided in those patients with high ischemic risk features requiring nondeferrable high hemorrhagic risk surgery between 1 and 6 months from PCI. ${ }^{5,18,19}$ As a matter of fact, maintaining antiplatelet therapy to minimize ischemic complications confers an increased risk of bleeding and need for transfusions, which are both determinants of poor prognosis, including higher mortality risk. ${ }^{4,25,31,32}$ In this setting, a strategy of temporary transition with a potent and effective intravenous antiplatelet agent with a predictable and safe interruption of platelet inhibition may represent an appropriate treatment option. ${ }^{4-6}$

Currently, the only intravenous antiplatelet agents available for clinical use and potentially usable for bridging include cangrelor and glycoprotein IIb/IIIa inhibitors (GPI). However, cangrelor is a more attractive agent for bridging. In fact, it has a very short half-life with rapid resumption of platelet function (within 60 minutes), it does not require renal dosing adjustments and has a specific dosing regimen for bridging identified from a dose-finding investigation aimed at achieving "thienopyridine-like" levels of platelet inhibition; moreover, cangrelor has been specifically tested in a prospective, randomized, double-blind, placebo-controlled trial of patients undergoing CABG. ${ }^{9,33}$ Conversely, the short-acting GPIs (i.e., eptifibatide and tirofiban) have a longer half-life and slower offset of action (i.e., 4-6 hours) compared with cangrelor, require renal dosing adjustments, and are used at the ACS dosing regimen given that there is no dedicated bridging dose. ${ }^{34}$ Moreover, GPIs are known to be associated with an increased risk of thrombocytopenia, particularly with prolonged infusion, a phenomenon associated with worse outcomes, including mortality. ${ }^{35}$ Overall, these pharmacologic characteristics enhance the risk of bleeding complications associated with GPI use which has never been tested for bridging in a randomized study. $9,31,36$ Accordingly, after a multidisciplinary assessment, we selected high thrombotic risk patients with previous PCI undergoing nondeferrable surgery. On one hand, these patients cannot safely interrupt oral antiplatelet therapy, on the other, they required a predictable interruption of platelet inhibition at the time of surgery to minimize blood loss. ${ }^{5,21}$ We tailored this strategy mainly for patients with a recent PCI or ACS with a 
clear indication to urgent cardiac or NCS with intermediate to high bleeding risk. Subsequently, we applied a prespecified bridging protocol with cangrelor infusion with a strict patient monitoring during the perioperative phase. ${ }^{22}$

To date, there is no randomized trial of bridging with cangrelor in NCS, and limited observational experience are available with heterogenous prescribing and monitoring practices, which may contribute to suboptimal outcomes. ${ }^{10-16}$ Likewise, data on bridging use of short-acting GPIs are heterogeneous as well with regard to type of surgery, inclusion criteria, time windows between stent implantation or ACS and surgery, and antiplatelet strategies during the perioperative period which may partly explain the reported variability in success rates. $^{37}$ Despite the inclusion of a similar miscellaneous of surgical procedures, we believed that the strength of our study is a standardize case by case approach evaluation to weight the ischemic and bleeding risk and the use of a bridging protocol with a close postoperative monitoring which could have had a positive impact on our study findings. ${ }^{19,20}$ Moreover, the possibility of restoration of a switch-on/switch-off infusion of a reversible antiplatelet agent (i.e., cangrelor) immediately after surgery when oral $\mathrm{P}_{2} \mathrm{Y}_{12}$ inhibiting therapy was temporarily not administrable, potentially allowed a safer monitoring in the postoperative phase when a careful balance between thrombotic and bleeding risk is particularly relevant. ${ }^{20}$ As a matter of fact, differently from previously reported worrisome increase in bleeding rates during bridging therapy with cangrelor, ${ }^{10,11,37}$ we showed no fatal/life-threatening bleedings or major blood loss requiring re-operation and a restrained mean hemoglobin drop. Additionally, one-third of patients experienced periprocedural BARC 3/GUSTO moderate bleeding events due to drainages output, mostly with a hemoglobin drop $<3 \mathrm{~g} / \mathrm{dL}$ and below the hemorrhages/transfusion rates (up 65\%) reported in previous observational studies on GPIs. ${ }^{37}$ We reported one cardiac death due to fatal ACS after cangrelor discontinuation 3 hours before surgery, which occurred after almost 2 months from PCI, while on aspirin. On one hand, these data underline the risk of DAPT discontinuation even beyond 1 month especially in patients treated with complex $\mathrm{PCI}$. In these patients, bridging with cangrelor up to 1 hour prior to surgical incision should be considered, even though the risk of thrombotic events might occur in perioperative phase, when cangrelor in suspended. On the other, previous studies on bridging therapy with cangrelor and eptifibatide had already highlighted such high ischemic risk profile reporting up to 3 to $6 \%$ rates of death or MI. ${ }^{37}$ To note, preoperative administration of tirofiban was associated with more favorable efficacy with the cost of increased bleeding complications, although different study designs and populations may partly or totally explain the variability in success rates reported in those studies. ${ }^{37}$ Undoubtedly, a strict selection of patient eligible for a bridging strategy needs to be fulfilled, excluding cases with lower bleeding risk profile to avoid overestimation of bleeding complications occurrence in predictable intermediate-to-low bleeding risk surgeries. ${ }^{20,38}$ Moreover, due to the well-known consistently elevated morbidity and mortality with no bridge therapy, ${ }^{2-4,28,29}$ more studies are warranted to support the efficacy and safety of our proposed standardized bridging strategy by identifying the patient population that would receive the maximum clinical benefit. Two multicenter observational registries will assess the impact of DAPT discontinuation on ischemic and bleeding events in stented patients referred to nondeferrable surgery. The MARS registry (ClinicalTrials.gov Identifier: NCT03981835) will study the current perioperative DAPT management strategies in the United States, including bridging, and outcome data after NCS. The MONET Italian study (ClinicalTrials.gov Identifier: NCT03445273) will evaluate outcomes after any type of surgery according to the actual application of the SAS2 indications and the clinical and angiographic risk of previous PCI. Furthermore, the ongoing randomized, double-blind, placebo-controlled MONET BRIDGE study (ClinicalTrials.gov Identifier: NCT03862651) aims to assess the efficacy and safety profile of a bridging strategy with cangrelor in patients who discontinue DAPT before surgery within 12 months from coronary stent implantation.

\section{Limitations}

Limitations of this study include the restricted number of patients and the heterogeneity between the enrolled patients and type of surgeries. However, prescribing and monitoring practices were standardized leading to more homogenous and consistent patient management which could have contributed to our overall favorable observations. Nevertheless, albeit within a certain time window, exact timing of initiation and discontinuation of cangrelor infusion was left at the discretion of the treating physician. Furthermore, any perioperative platelet function test and transfusion was suggested. Despite the lack of evidence to guide the prophylactic use of platelet transfusions before major surgery, they should be considered according to the threshold of platelet count and in case of severe thrombocytopenia or critical bleeding. ${ }^{39,40}$ Moreover, due to a large individual variation in the magnitude and duration of the antiplatelet effect, platelet function testing may be considered to help guide timing of starting cangrelor infusion after a $\mathrm{P}_{2} \mathrm{Y}_{12}$ discontinuation, especially in case of planned cardiac surgery. ${ }^{5,22}$ Finally, data interpretation warrants caution due to the lack of a control group. Despite the abovementioned limitations, we think that our paper may add useful information to the current literature, reporting for the first time a strategy with a standardize case by case approach evaluation to weight the ischemic and bleeding risk and the use of a bridging protocol with a close postoperative monitoring. Moreover, this data might help to identify the patient population that would receive maximum benefit from bridging antiplatelet therapy, determine optimal administration strategy, monitoring therapy, and management of adverse events to delineate large prospective studies which are required in such challenging setting. ${ }^{41}$

\section{Conclusion}

Perioperative antiplatelet bridging therapy with cangrelor is a feasible approach for stented patients at high thrombotic risk referred to nondeferrable intermediate-high bleeding 
risk surgery requiring DAPT discontinuation. Larger studies are warranted to support the safety of this strategy.

\section{Conflict of Interest}

R.R. reports payment as an individual for consulting fee or honorarium from Eli Lilly and Co., and Daiichi Sankyo, Inc, Astra Zeneca, Bayer, and Pfizer. G.M. reports honoraria for lectures from Eli Lilly and Co., and Daiichi Sankyo, Inc, Astra Zeneca, St. Jude Medical, and Abbott Vascular. D.J.A. declares that he has received consulting fees or honoraria from Amgen, Aralez, AstraZeneca, Bayer, Biosensors, Boehringer Ingelheim, Bristol-Myers Squibb, Chiesi, Daiichi-Sankyo, Eli Lilly, Haemonetics, Janssen, Merck, PhaseBio, PLx Pharma, Pfizer, Sanofi, and the Medicines Company and has received payments for participation in review activities from CeloNova and St. Jude Medical. D. J.A. also declares that his institution has received research grants from Amgen, AstraZeneca, Bayer, Biosensors, CeloNova, CSL Behring, Daiichi-Sankyo, Eisai, Eli Lilly, Gilead, Idorsia, Janssen, Matsutani Chemical Industry Co., Merck, Novartis, Osprey Medical, and Renal Guard Solutions. The other authors report no conflict of interest.

\section{References}

1 Capodanno D, Alfonso F, Levine GN, Valgimigli M, Angiolillo DJ. ACC/AHA versus ESC guidelines on dual antiplatelet therapy: JACC guideline comparison. J Am Coll Cardiol 2018;72(23 Pt A):2915-2931

2 Rossini R, Musumeci G, Capodanno D, et al. Perioperative management of oral antiplatelet therapy and clinical outcomes in coronary stent patients undergoing surgery. Results of a multicentre registry. Thromb Haemost 2015;113(02):272-282

3 Schouten O, van Domburg RT, Bax JJ, et al. Noncardiac surgery after coronary stenting: early surgery and interruption of antiplatelet therapy are associated with an increase in major adverse cardiac events. J Am Coll Cardiol 2007;49(01):122-124

4 Capodanno D, Angiolillo DJ. Management of antiplatelet therapy in patients with coronary artery disease requiring cardiac and noncardiac surgery. Circulation 2013;128(25):2785-2798

5 Valgimigli M, Bueno H, Byrne RA, et al;ESC Scientific Document Group ESC Committee for Practice Guidelines (CPG) ESC National Cardiac Societies. 2017 ESC focused update on dual antiplatelet therapy in coronary artery disease developed in collaboration with EACTS: the task force for dual antiplatelet therapy in coronary artery disease of the European Society of Cardiology (ESC) and of the European Association for Cardio-Thoracic Surgery (EACTS). Eur Heart J 2018;39(03):213-260

6 Rossini R, Angiolillo DJ, Musumeci G, et al. Antiplatelet therapy and outcome in patients undergoing surgery following coronary stenting: results of the surgery after stenting registry. Catheter Cardiovasc Interv 2017;89(01):E13-E25

7 Bhatt DL, Stone GW, Mahaffey KW, et al;CHAMPION PHOENIX Investigators. Effect of platelet inhibition with cangrelor during PCI on ischemic events. N Engl J Med 2013;368(14):1303-1313

8 Angiolillo DJ, Schneider DJ, Bhatt DL, et al. Pharmacodynamic effects of cangrelor and clopidogrel: the platelet function substudy from the cangrelor versus standard therapy to achieve optimal management of platelet inhibition (CHAMPION) trials. J Thromb Thrombolysis 2012;34(01):44-55

9 Angiolillo DJ, Firstenberg MS, Price MJ, et al;BRIDGE Investigators. Bridging antiplatelet therapy with cangrelor in patients undergoing cardiac surgery: a randomized controlled trial. JAMA 2012; 307(03):265-274
10 Bowman S, Gass J, Weeks P. Antiplatelet therapy bridging with cangrelor in patients with coronary stents: a case series. Ann Pharmacother 2019;53(02):171-177

11 Van Tuyl JS, Newsome AS, Hollis IB. Perioperative bridging with glycoprotein IIb/IIIa inhibitors versus cangrelor: balancing efficacy and safety. Ann Pharmacother 2019;53(07):726-737

12 Stern G, Rimsans J, Qamar A, Vaduganathan M, Bhatt DL. Contemporary parenteral antiplatelet bridging strategies: a single-centre real-world experience at a tertiary care centre. EuroIntervention 2018;14(03):e333-e335

13 Kabadi RA, Danelich IM, Entwistle JW III, et al. Use of cangrelor as a bridge to left ventricular assist device implantation in a patient with a recent drug-eluting stent who developed acute tirofibanrelated thrombocytopenia. Pharmacotherapy 2019;39(04): 521-525

14 Calnan MW, Crawford AN. A case report of cangrelor bridge therapy for a diagnostic bronchoscopy with biopsy. J Pharm Pract 2020;33(02):231-235

15 Cahoon WD Jr, Oswalt AK, Francis KE, Magee LC, Lowe DK. Cangrelor bridge therapy for gastroduodenal biopsy. J Pharm Pract 2017;30(02):270-273

16 Laehn SJ, Feih JT, Saltzberg MT, Garner Rinka JR. Pharmacodynamic-guided cangrelor bridge therapy for orthotopic heart transplant. J Cardiothorac Vasc Anesth 2019;33(04):1054-1058

17 Kristensen SD, Knuuti J, Saraste A, et al;Authors/Task Force Members. 2014 ESC/ESA Guidelines on non-cardiac surgery: cardiovascular assessment and management: The Joint Task Force on non-cardiac surgery: cardiovascular assessment and management of the European Society of Cardiology (ESC) and the European Society of Anaesthesiology (ESA). Eur Heart J 2014;35 (35):2383-2431

18 Levine GN, Bates ER, Bittl JA, et al. 2016 ACC/ AHA guideline focused update on duration of dual antiplatelet therapy in patients with coronary artery disease: a report of the American College of Cardiology/American Heart Association Task Force on Clinical Practice Guidelines. J Am Coll Cardiol 2016;68(10): 1082-1115

19 Neumann FJ, Sousa-Uva M, Ahlsson A, et al;ESC Scientific Document Group. 2018 ESC/EACTS Guidelines on myocardial revascularization. Eur Heart J 2019;40(02):87-165

20 Rossini R, Musumeci G, Visconti LO, et al;Italian Society of Invasive Cardiology (SICI-GISE) Italian Association of Hospital Cardiologists (ANMCO) Italian Society for Cardiac Surgery (SICCH) Italian Society of Vascular and Endovascular Surgery (SICVE) Italian Association of Hospital Surgeons (ACOI) Italian Society of Surgery (SIC) Italian Society of Anaesthesia and Intensive Care Medicine (SIAARTI) Lombard Society of Surgery (SLC) Italian Society of Maxillofacial Surgery (SICMF) Italian Society of Reconstructive Plastic Surgery and Aesthetics (SICPRE) Italian Society of Thoracic Surgeons (SICT) Italian Society of Urology (SIU) Italian Society of Orthopaedics and Traumatology (SIOT) Italian Society of Periodontology (SIdP) Italian Federation of Scientific Societies of Digestive System Diseases Lombardia (FISMAD) Association of Obstetricians Gynaecologists Italian Hospital Lombardia (AOGOI) Society of Ophthalmology Lombardia (SOL). Perioperative management of antiplatelet therapy in patients with coronary stents undergoing cardiac and non-cardiac surgery: a consensus document from Italian cardiological, surgical and anaesthesiological societies. EuroIntervention 2014;10(01):38-46

21 Rossini R, Tarantini G, Musumeci G, et al;Italian Society of Interventional Cardiology (SICI-GISE) Italian Society for the Study of Haemostasis and Thrombosis (SISET) Italian Society of Anesthesia and Intensive Care Medicine (SIAARTI) Italian Society of Surgery (SIC) Italian Society for Cardiac Surgery (SICCH) Italian Society of Vascular and Endovascular Surgery (SICVE) Italian Society of Urology (SIU) Italian Orthopaedic Society (SIOT) Italian Society of Thoracic Surgeons (SICT) Italian Federation of Scientific Societies of Digestive System Diseases (FISMAD) Italian Society of 
Digestive Endoscopy (SIED) Italian Association of Hospital Gastroenterology and Digestive Endoscopy (AIGO) Italian Association of Gastroenterology and Digestive Endoscopy (SIGE) Italian Society of Maxillofacial Surgery (SICMF) Italian Society of Reconstructive Plastic Surgery and Aesthetics (SICPRE) Italian Society of Gynecology and Obstetrics (SIGO) Italian Society of Neurosurgery (SINch) Italian Association of Hospital Pulmonologist (AIPO) Italian Society of Periodontology (SIdP) Italian Society of Ophthalmology (SOI) Italian Association of Hospital Otorhinolaryngologist (AOOI) Italian Association of Hospital Surgeons (ACOI) Association of Obstetricians Gynecologists Italian Hospital (AOGOI). A multidisciplinary approach on the perioperative antithrombotic management of patients with coronary stents undergoing surgery: surgery after stenting 2. JACC Cardiovasc Interv 2018;11(05):417-434

22 Angiolillo DJ, Rollini F, Storey RF, et al. International expert consensus on switching platelet $\mathrm{P}_{2} \mathrm{Y}_{12}$ receptor-inhibiting therapies. Circulation 2017;136(20):1955-1975

23 Franchi F, Rollini F, Muñiz-Lozano A, Cho JR, Angiolillo DJ. Cangrelor: a review on pharmacology and clinical trial development. Expert Rev Cardiovasc Ther 2013;11(10):1279-1291

24 Cutlip DE, Windecker S, Mehran R, et al;Academic Research Consortium. Clinical end points in coronary stent trials: a case for standardized definitions. Circulation 2007;115(17):2344-2351

25 Vaduganathan M, Harrington RA, Stone GW, et al. Short- and longterm mortality following bleeding events in patients undergoing percutaneous coronary intervention: insights from four validated bleeding scales in the CHAMPION trials. EuroIntervention 2018; 13(15):e1841-e1849

26 Conte MS, Bradbury AW, Kolh P, et al;GVG Writing Group for the Joint Guidelines of the Society for Vascular Surgery (SVS), European Society for Vascular Surgery (ESVS), and World Federation of Vascular Societies (WFVS). Global vascular guidelines on the management of chronic limb-threatening ischemia. Eur J Vasc Endovasc Surg 2019;58(1S):S1-109.e33

27 Begic D, Mujicic E, Coric J, Zec SL, Zunic L. Analysis of the blood consumption for surgical programs. Med Arh 2016;70(04):248-251

28 Savonitto S, Caracciolo M, Cattaneo M, DE Servi S. Management of patients with recently implanted coronary stents on dual antiplatelet therapy who need to undergo major surgery. J Thromb Haemost 2011;9(11):2133-2142

29 Albaladejo P, Marret E, Samama CM, et al. Non-cardiac surgery in patients with coronary stents: the RECO study. Heart 2011;97 (19):1566-1572
30 Egholm G, Kristensen SD, Thim T, et al. Risk associated with surgery within 12 months after coronary drug-eluting stent implantation. J Am Coll Cardiol 2016;68(24):2622-2632

31 van Kuijk JP, Flu WJ, Schouten O, et al. Timing of noncardiac surgery after coronary artery stenting with bare metal or drugeluting stents. Am J Cardiol 2009;104(09):1229-1234

32 Brilakis ES, Banerjee S, Berger PB. Perioperative management of patients with coronary stents. J Am Coll Cardiol 2007;49(22): $2145-2150$

33 Dargham BB, Baskar A, Tejani I, et al. Intravenous antiplatelet therapy bridging in patients undergoing cardiac or non-cardiac surgery following percutaneous coronary intervention. Cardiovasc Revasc Med 2019;20(09):805-811

34 Muñiz-Lozano A, Rollini F, Franchi F, Angiolillo DJ. Update on platelet glycoprotein IIb/IIIa inhibitors: recommendations for clinical practice. Ther Adv Cardiovasc Dis 2013;7(04):197-213

35 Groves EM, Bhatt DL, Steg PG, et al. Incidence, predictors, and outcomes of acquired thrombocytopenia after percutaneous coronary intervention: a pooled, patient-level analysis of the CHAMPION Trials (cangrelor versus standard therapy to achieve optimal management of platelet inhibition). Circ Cardiovasc Interv 2018; 11(04):e005635

36 De Servi S, Morici N, Boschetti E, et al. Bridge therapy or standard treatment for urgent surgery after coronary stent implantation: analysis of 314 patients. Vascul Pharmacol 2016;80:85-90

37 Morici N, Moja L, Rosato V, et al. Bridge with intravenous antiplatelet therapy during temporary withdrawal of oral agents for surgical procedures: a systematic review. Intern Emerg Med 2014;9(02):225-235

38 Chew DP, Junbo G, Parsonage W, et al;Perceived Risk of Ischemic and Bleeding Events in Acute Coronary Syndrome Patients (PREDICT) Study Investigators. Perceived risk of ischemic and bleeding events in acute coronary syndromes. Circ Cardiovasc Qual Outcomes 2013;6(03):299-308

39 Estcourt LJ, Birchall J, Allard S, et al;British Committee for Standards in Haematology. Guidelines for the use of platelet transfusions. Br J Haematol 2017;176(03):365-394

40 Makris M, Van Veen JJ, Tait CR, Mumford AD, Laffan MBritish Committee for Standards in Haematology. Guideline on the management of bleeding in patients on antithrombotic agents. Br J Haematol 2013;160(01):35-46

41 Bhattad VB, Gaddam S, Lassiter MA, et al. Intravenous cangrelor as a peri-procedural bridge with applied uses in ischemic events. Ann Transl Med 2019;7(17):408 